\title{
HOLOMORPHIC KERNELS AND COMMUTING OPERATORS
}

\author{
AMEER ATHAVALE
}

\begin{abstract}
Necessary and sufficient conditions in terms of operator polynomials are obtained for an $m$-tuple $T=\left(T_{1}, \ldots, T_{m}\right)$ of commuting bounded linear operators on a separable Hilbert space $\mathscr{H}$ to extend to an $\dot{m}$-tuple $S=\left(S_{1}, \ldots, S_{m}\right)$ of operators on some Hilbert space $\mathscr{K}$, where each $S_{i}$ is realized as a *-representation of the adjoint of a multiplication operator on the tensor product of a special type of functional Hilbert spaces. Also, necessary and sufficient conditions in terms of operator polynomials are obtained for $T$ to have a commuting normal extension.
\end{abstract}

0. Introduction. In this paper, some results in [1 and 2] for a single bounded linear operator $T$ on a separable Hilbert space $\mathscr{H}$ are generalized to $m$ commuting operators on $\mathscr{H}$. In [1], Agler introduces a special class of functional Hilbert spaces $\mathscr{M}$ and describes conditions under which an operator $T$ on $\mathscr{H}$ extends to $M^{*(\infty)}$, where $M$ denotes the multiplication operator on $\mathscr{M}$, and $M^{*(\infty)}$ denotes the countable direct sum of $M^{*}$ with itself. Special cases of spaces $\mathscr{M}$, of which the classical Hardy space is the prototype, are considered in $[\mathbf{1}, 2]$. The relevant conditions for the kind of extension of $T$ referred to above are expressed in terms of the positivity of certain operator polynomials involving $T$ and $T^{*}$. These conditions are closely related to the reproducing kernel associated with the space $\mathscr{M}$. In [2], necessary and sufficient conditions are also given for a contraction $T$ to be subnormal. These conditions are really the requirement that a certain sequence of polynomials in $T$ and $T^{*}$ be positive. It is natural to seek generalizations of these results to $m$ commuting operators $T_{1}, \ldots, T_{m}$ on $\mathscr{H}$. An appropriate model for this generalization is obtained by constructing a finite tensor product of spaces $\mathscr{M}$ and exploiting the well-known fact that the reproducing kernel of such a tensor product is the product of the reproducing kernels of the individual spaces. Suitable modifications of the reproducing kernels which render them holomorphic on the unit polydisc can be used to describe analogous extension results for $T=\left(T_{1}, \ldots, T_{m}\right)$. The question of $T$ having a commuting normal extension $N=\left(N_{1}, \ldots, N_{m}\right)$ turns out to have a direct link with the multi-dimensional Hausdorff Moment Problem from the theory of probability.

Received by the editors March 21, 1986.

1980 Mathematics Subject Classification. Primary 47A20; Secondary 47B20, 47B38.

Key words and phrases. Positive definite, regular analytic model atom, kernel, tensor product, commuting normal extension, *-dilation.

This work constitutes part of the author's Ph. D. dissertation written at Indiana University under the direction of John B. Conway. 
$\$ 1$ fixes some notation and introduces the idea of a positive definite matrix of functions defined on the cartesian product of two unit polydiscs. $\$ 2$ develops the necessary theory of holomorphic kernels in the setting of the tensor product of a finite number of functional Hilbert spaces and derives general results pertaining to extensions of $T$. $\S 3$ presents applications of these results to several concrete examples. $\S 4$ deals with the existence of a commuting normal extension for $T$ and concludes with some general discussion.

1. Preliminaries. The set of bounded linear operators on a Hilbert space $\mathscr{H}$ will be denoted by $\mathscr{B}(\mathscr{H}) ; \mathscr{H}^{(n)}$ will denote the direct sum of $\mathscr{H}$ with itself $n$ times. An $m$-tuple $\left(b_{1}, \ldots, b_{m}\right)$ of commuting elements in a $C^{*}$-algebra will be denoted by $b$, while $b^{*}$ will stand for $\left(b_{1}^{*}, \ldots, b_{m}^{*}\right)$. Similarly, any $m$-tuple $\left(z_{1}, \ldots, z_{m}\right)$ of complex numbers will be abbreviated to $z$, with $z^{*}$ having the obvious meaning. For any $m$-tuple $\left(s_{1}, \ldots, s_{m}\right)$ of nonnegative integers, $b^{s}$ will denote $b_{1}^{s_{1}} b_{2}^{s_{2}} \cdots b_{m}^{s_{m}}$, while $z^{s}$ will stand for $z_{1}^{s_{1}} z_{2}^{s_{2}} \cdots z_{m}^{s_{m}}$. For any subset $A$ of the complex plane $\mathbf{C}, A^{*}$ and $A^{m}$ will respectively mean $\left\{z \in \mathbf{C}: z^{*} \in A\right\}$ and the cartesian product of $A$ with itself $m$ times. For any open set $G$ in $\mathbf{C}^{m}, H(G)$ will be the set of holomorphic functions on $G$ and $L^{2}(G, \mu)$ will be the square integrable functions with respect to measure $\mu$ on $G$. In general, various abbreviations used in the paper will be clear from the context in which they appear. All the Hilbert spaces occurring below are separable. The arguments here parallel those in $[\mathbf{1}, \mathbf{2}]$.

We begin by introducing a functional calculus for $\left(b, b^{*}\right)$, where for $1 \leqslant i \leqslant m$, the spectrum $\sigma\left(b_{i}\right)$ of $b_{i}$ is contained in some open disc $G_{i}$ in $\mathbf{C}$, with center at $z_{i}=0$. If $f \in H\left(\left(G_{1} \times \cdots \times G_{m}\right)^{2}\right)$, define

$$
\text { (1) } \begin{aligned}
& f\left(b, b^{*}\right)=\left(\frac{1}{2 \pi i}\right)^{2 m} \int_{\gamma_{m}^{*}} \cdots \int_{\gamma_{1}^{*}} \int_{\gamma_{m}} \cdots \int_{\gamma_{1}} f(z, w)\left(w_{m}-b_{m}^{*}\right)^{-1} \\
& \cdots\left(w_{1}-b_{1}^{*}\right)^{-1}\left(z_{m}-b_{m}\right)^{-1} \\
& \cdots\left(z_{1}-b_{1}\right)^{-1} d z_{1} \cdots d z_{m} d w_{1} \cdots d w_{m},
\end{aligned}
$$

where $\gamma_{i}$ is any finite system of Jordan arcs surrounding $\sigma\left(b_{i}\right)$ and lying in $G_{i}$. We note in particular the following two consequences of the above definition.

(a) If $p(z, w)=\sum_{s, t} c_{s t} z^{s} w^{t}$ is a polynomial in $2 m$ complex variables $(z, w)$, then

$$
p\left(b, b^{*}\right)=\sum_{s, t} c_{s t} b^{* t} b^{s} .
$$

(b) If $f_{1}, f_{2} \in H\left(G_{1} \times \cdots \times G_{m}\right)$ and $f \in H\left(\left(G_{1} \times \cdots \times G_{m}\right)^{2}\right)$, then

$$
f_{2}\left(b^{*}\right) f\left(b, b^{*}\right) f_{1}(b)=g\left(b, b^{*}\right),
$$

where $g(z, w)=f_{1}(z) f_{2}(w) f(z, w)$.

Relation (3) can be checked easily by verifying it first for powers of $z$ and $w$ and then noting that any $f \in H\left(\left(G_{1} \times \cdots \times G_{m}\right)^{2}\right)$ can be expressed as

$$
f(z, w)=\sum_{s, t} a_{s t} z^{s} w^{t}
$$


where the series on the right-hand side converges uniformly to $f$ on compact subsets of $\left(G_{1} \times \cdots \times G_{m}\right)^{2}[9]$.

Definition 1.1. If $D$ is the open unit disc in $\mathbf{C}$, then an $n \times n$ matrix $\left[g_{i j}\right]$ of functions defined on $D^{m} \times D^{m}$ is positive definite if for all positive integers $p$, all vectors $C_{r}$ in $\mathbf{C}^{(n)}$, and all points $\lambda^{(r)}$ in $D^{m}(1 \leqslant r \leqslant p)$,

$$
\sum_{1 \leqslant r, s \leqslant p}\left\langle G_{r s} C_{r}, C_{s}\right\rangle_{\mathbf{C}^{(n)}} \geqslant 0
$$

where $G_{r s}$ is the $n \times n$ matrix $\left[g_{i j}\left(\lambda^{(r)}, \lambda^{(s)}\right)\right]$ and where $\langle\cdot, \cdot\rangle_{\mathbf{C}^{(n)}}$ denotes the inner product in $\mathbf{C}^{(n)}$.

Lemma 1.2. Let $\left[g_{i j}\right]$ be positive definite as in Definition 1.1. Also let $g_{i j}\left(z^{*}, w\right)$ be holomorphic on $D^{m} \times D^{m}$ for $1 \leqslant i, j \leqslant n$. Then for any $r$ such that $0<r<1$, there exist functions $f_{i l}, 1 \leqslant i \leqslant n, 1 \leqslant l$, defined and holomorphic on $(r D)^{m}$ such that

$$
g_{i j}(z, w)=\sum_{l=1}^{\infty} f_{j l}^{*}(z) f_{i l}(w)
$$

for $1 \leqslant i, j \leqslant n$, and where the series on the right converges uniformly on compact subsets of $(r D)^{m} \times(r D)^{m}$.

Proof. Define $A_{i j}$ on $\left.L^{2}\left((r D)^{m} \text {, (Area) }\right)^{m}\right)$ by

$$
A_{i j}(f)(w)=\int_{(r D)^{m}} g_{i j}(z, w) f(z) d(\text { Area })^{m}(z) .
$$

Further, let $A=\left[A_{i j}\right] \in \mathscr{B}\left(\left(L^{2}\left((r D)^{m},(\text { Area })^{m}\right)\right)^{(n)}\right)$. Since $g_{i j}$ is bounded on $(r D)^{m} \times(r D)^{m}, A$ is a compact operator. Using the fact that $\left[g_{i j}\right]$ is positive definite, it is easy to see that $A$ is a positive operator. If $\left\{t_{l}\right\}$ is the sequence of nonzero eigenvalues of $A$ and $h_{l}=\oplus_{i=1}^{n} h_{i l}$, the corresponding eigenfunctions, then (6) holds for $f_{i l}=\sqrt{t_{l}} h_{i l}$, as can be seen by identifying

$$
\mathcal{N}_{n}\left(L^{2}\left((r D)^{m} \times(r D)^{m},(\text { Area })^{m} \times(\text { Area })^{m}\right)\right)
$$

with $\mathscr{C}_{2}\left(\left(L^{2}\left((r D)^{m},(\text { Area })^{m}\right)\right)^{(n)}\right)$, where the former denotes the Hilbert space of $n \times n$ matrices with entries $p_{i j}$ from $L^{2}\left((r D)^{m} \times(r D)^{m},(\text { Area })^{m} \times(\text { Area })^{m}\right)$ and with the inner product

$$
\begin{aligned}
& \left\langle\left[p_{i j}\right],\left[q_{i j}\right]\right\rangle \\
& \quad=\sum_{1 \leqslant i, j \leqslant n} \int_{(r D)^{m} \times(r D)^{m}} p_{i j}(z, w) q_{i j}^{*}(z, w) d(\text { Area })^{m} \times(\text { Area })^{m}(z, w) ;
\end{aligned}
$$

and the latter denotes the Schmidt class of operators on $\left(L^{2}\left((r D)^{m},(\text { Area })^{m}\right)\right)^{(n)}$. (This is indeed the matricial analog of Theorem 2.4.4 in [10].) Note that the series in (6) converges in the norm $\|\cdot\|$ of $\left.L^{2}\left((r D)^{m} \times(r D)^{m} \text {, (Area) }\right)^{m} \times(\text { Area })^{m}\right)$. Since $h_{l}$ is an eigenfunction corresponding to the nonzero eigenvalue $t_{l}$, using (7) the functions $f_{i l}$ are seen to be holomorphic on $(r D)^{m}$. That the series in (6) converges uniformly on compact subsets of $(r D)^{m} \times(r D)^{m}$ follows by noting that if $K_{1} \times \cdots \times K_{2 m}$ is any compact subset of $(r D)^{m} \times(r D)^{m}$, then for any $f \in$ $H\left((r D)^{m} \times(r D)^{m}\right)$, and any point $z_{0}$ of $K_{1} \times \cdots \times K_{2 m}$,

$$
\|f\| \geqslant \pi^{m}\left(r_{1} r_{2} \cdots r_{2 m}\right)\left|f\left(z_{0}\right)\right|
$$

where $r_{i}$ is the distance of $K_{i}$ from the boundary of $r D$. 
DEFINITION 1.3. Let $T$ be a tuple of $m$ commuting operators on a Hilbert space $\mathscr{H}$. We say $T$ extends to $S$ if there exists a Hilbert space $\mathscr{K}, S_{i} \in \mathscr{B}(\mathscr{K})(1 \leqslant i \leqslant m)$, and an isometry $V$ from $\mathscr{H}$ into $\mathscr{K}$ such that Range $V$ is invariant for each $S_{i}$ and $T_{i}=V^{*} S_{i} V$ for every $i$. (It follows then that $p\left(T, T^{*}\right)=V^{*} p\left(S, S^{*}\right) V$ for any $p\left(T, T^{*}\right), p\left(S, S^{*}\right)$ as in (2).)

THEOREM 1.4. Let $B$ be $a C^{*}$-algebra with identity 1 and let $b$ be a tuple of $m$ commuting elements in $B$. If $T$ is a tuple of $m$ commuting elements in $\mathscr{B}(\mathscr{H})$, where $\mathscr{H}$ is a Hilbert space, then the following are equivalent.

(i) There exist a Hilbert space $\mathscr{K}$ and $a *$-representation $\pi: B \rightarrow \mathscr{B}(\mathscr{K})$ with $\pi(1)=1 ;$ and $T$ extends to $\pi(b)=\left(\pi\left(b_{1}\right), \ldots, \pi\left(b_{m}\right)\right)$.

(ii) For any positive integer $n$ and $n^{2}$ polynomials $p_{i j}$ in $2 m$ complex variables,

$$
\left[p_{i j}\left(b, b^{*}\right)\right] \geqslant 0 \text { in } \mathscr{N}_{n}(B) \text { implies }\left[p_{i j}\left(T, T^{*}\right)\right] \geqslant 0 \text { in } \mathscr{B}\left(\mathscr{H}^{(n)}\right),
$$

where $\mathscr{N}_{n}(B)$ denotes the class of $n \times n$ matrices with entries from $B$. (Given $B$, $\mathscr{N}_{n}(B)$ is equipped with a unique $C^{*}$-norm.)

The proof of Theorem 1.4 follows the same lines as in Theorem 1.5 in [1] and uses the Stinespring Representation Theorem and the Arveson Extension Theorem [4, Theorem 1.2.3]. The proof is omitted.

2. Kernel functions and kernels. We now introduce a special class of functional Hilbert spaces [1].

Definition 2.1. An analytic model atom $\mathscr{M}$ over $D$ is a Hilbert space of analytic functions on $D$ satisfying the following properties.

(i) For any $\lambda \in D, f, g \in \mathscr{M}$, and $\alpha, \beta \in \mathbf{C},(\alpha f+\beta g)(\lambda)=\alpha f(\lambda)+\beta g(\lambda)$.

(ii) For every $\lambda \in D$, there is a constant $c_{\lambda}$ such that $|f(\lambda)| \leqslant c_{\lambda}\|f\|_{\mathscr{M}}$ for $f$ in $\mathscr{M}$. Here $\|\cdot\|_{\mathscr{M}}$ denotes the norm induced by the inner product $\langle\cdot, \cdot\rangle_{\mathscr{M}}$ of $\mathscr{M}$.

(iii) If $f$ is holomorphic on a neighborhood of the closure $\bar{D}$ of $D$, then $f \in \mathscr{M}$.

(iv) The map $M$ defined on $\mathscr{M}$ by $(M f)(z)=z f(z), f \in \mathscr{M}$, is a bounded operator on $\mathscr{M}$.

Note that by virtue $v_{i}$ (ii) above, there exists for any $\lambda \in D$ a function $\kappa_{\lambda}$ in $\mathscr{M}$ such that $f(\lambda)=\left\langle f, \kappa_{\lambda}\right\rangle_{\mathscr{M}}$ for any $f \in \mathscr{M}$. The kernel functions $\kappa_{\lambda}$ give rise to the kernel $\kappa(\lambda, \mu)$ of $\mathscr{M}$ defined by $\kappa(\lambda, \mu)=\left\langle\kappa_{\mu^{*}}, \kappa_{\lambda}\right\rangle_{\mathscr{M}}=\kappa_{\mu^{*}}(\lambda)$. Note that $\kappa(\lambda, \mu) \in$ $H\left(D^{2}\right)$.

Definition 2.2. An analytic model atom $\mathscr{M}$ over $D$ is called regular if it satisfies the following properties.

(i) Polynomials in $z$ are dense in $\mathscr{M}$.

(ii) The kernel $\kappa(\lambda, \mu)$ does not vanish anywhere on $D^{2}$, and $\kappa$ is symmetric, that is $\kappa(\lambda, \mu)=\kappa(\mu, \lambda)$.

(iii) The operator $\lambda-M \in \mathscr{B}(\mathscr{M})$ is Fredholm for every $\lambda \in D$.

Let $\mathscr{M}_{1}, \ldots, \mathscr{M}_{m}$ be $m$ regular analytic model atoms over $D$ with kernels $\kappa_{1}, \ldots, \kappa_{m}$ respectively. The tensor product $\mathscr{M}=\mathscr{M}_{1} \otimes \cdots \otimes \mathscr{M}_{m}$ is also a functional Hilbert space [3] with the "kernel" $\kappa$ given by

$$
\kappa(\lambda, \mu)=\kappa_{1}\left(\lambda_{1}, \mu_{1}\right) \kappa_{2}\left(\lambda_{2}, \mu_{2}\right) \cdots \kappa_{m}\left(\lambda_{m}, \mu_{m}\right) .
$$


The kernel $\kappa$ is related to the "kernel functions" $\kappa_{\lambda}=\kappa_{\lambda_{1}} \otimes \cdots \otimes \kappa_{\lambda_{m}}$ by $\kappa(\lambda, \mu)=$ $\left\langle\kappa_{\mu^{*}}, \kappa_{\lambda}\right\rangle_{\mathscr{M}}$. If $M_{i}$ denotes multiplication by $z$ on $\mathscr{M}_{i}$, then define a corresponding operator $\underline{M}_{i}$ on $\mathscr{M}_{1} \otimes \cdots \otimes \mathscr{M}_{m}$ by $\underline{M}_{i}=1 \otimes \cdots \otimes M_{i} \otimes \cdots \otimes 1$. We use $\underline{M}$ to denote $\left(\underline{M}_{1}, \ldots, \underline{M}_{m}\right)$. Note that $\sigma\left(\underline{M}_{i}\right)=\sigma\left(M_{i}\right)=\bar{D}$.

Lemma 2.3. Let $\mathscr{M}=\mathscr{M}_{1} \otimes \cdots \otimes \mathscr{M}_{m}$, where each $M_{i}$ is a regular analytic model atom over $D$. If $p(z, w)$ is any polynomial, then

$$
\left\langle p\left(\underline{M}^{*}, \underline{M}\right) \kappa_{\lambda}, \kappa_{\mu}\right\rangle_{\mathscr{M}}=p\left(\lambda^{*}, \mu\right) \kappa\left(\lambda^{*}, \mu\right) .
$$

Proof. Use the fact that for any $f \in \mathscr{M}$, it follows that $\left\langle f, \underline{M}_{j}^{*} k_{\lambda}\right\rangle_{\mathscr{M}}=$ $\left\langle\underline{M}_{j} f, k_{\lambda}\right\rangle_{\mathscr{M}}=\lambda_{j} f(\lambda)=\lambda_{j}\left\langle f, k_{\lambda}\right\rangle_{\mathscr{M}}=\left\langle f, \lambda_{j}^{*} \kappa_{\lambda}\right\rangle_{\mathscr{M}}$.

LeMma 2.4. Let $\mathscr{M}$ be as in Lemma 2.3. Then for any positive integer $n$ and $n^{2}$ polynomials $p_{i j},\left[p_{i j}\left(\underline{M}^{*}, \underline{M}\right)\right] \geqslant 0$ in $\mathscr{B}\left(\mathscr{M}^{(n)}\right)$ if and only if $\left[p_{i j}\left(z^{*}, w\right) \kappa\left(z^{*}, w\right)\right]$ is positive definite.

Proof. Use Lemma 2.3 and argue as in Proposition 2.5 in [1].

We now arrive at one of the principal results of the paper.

THEOREM 2.5. Let $\mathscr{M}$ be the Hilbert space with kernel $\kappa$ obtained by tensoring $m$ regular analytic model atoms $\mathscr{M}_{1}, \mathscr{M}_{2}, \ldots, \mathscr{M}_{m}$ over $D$. Let, for any extended integer $n(0<n \leqslant \infty), M_{i}^{*(n)}$ denote the direct sum of $M_{i}^{*}$ with itself $n$ times, where $M_{i}$ denotes multiplication by $z$ on $\mathscr{M}_{i}$. Let $\mathscr{H}$ be a Hilbert space and let $T$ be a tuple of $m$ commuting operators on $\mathscr{H}$ such that $\sigma\left(T_{i}\right) \subset D(1 \leqslant i \leqslant m)$. If $\frac{1}{\kappa}\left(T, T^{*}\right) \geqslant 0$, then there exist a Hilbert space $\mathscr{K}$ and a *-representation $\pi: \mathscr{B}(\mathscr{M}) \rightarrow \mathscr{B}(\mathscr{K})$ with $\pi(1)=1$ such that $T$ extends to $\pi\left(\underline{M}^{*}\right)$ and where $\pi\left(\underline{M}_{i}^{*}\right)$ is unitarily equivalent to $M_{i}^{*\left(n_{i}\right)} \oplus W_{i}$ with $\sigma\left(W_{i}\right) \subset \partial D$, the boundary of $D$, and $0<n_{i} \leqslant \infty(1 \leqslant i \leqslant m)$. (One of the summands in $M_{i}^{*\left(n_{i}\right)} \oplus W_{i}$ may be absent.)

Proof. Assume $\frac{1}{\kappa}\left(T, T^{*}\right) \geqslant 0$. Using Lemmas 1.2 and 2.4 and arguing as in Theorem 2.3 of [1], it is not difficult to show that for any positive integer $n$ and $n^{2}$ polynomials $p_{i j}, \quad\left[p_{i j}\left(\underline{M}^{*}, \underline{M}\right)\right] \geqslant 0$ in $\mathscr{B}\left(\mathscr{M}^{(n)}\right)$ implies $\left[p_{i j}\left(T, T^{*}\right)\right] \geqslant 0$ in $\mathscr{B}\left(\mathscr{H}^{(n)}\right)$. By Theorem 1.4, there exist a Hilbert space $\mathscr{K}$ and a $*$-representation $\pi$ : $\mathscr{B}(\mathscr{M}) \rightarrow \mathscr{B}(\mathscr{K})$ with $\pi(1)=1$ such that $T$ extends to $\pi\left(\underline{M}^{*}\right)$. (Note the identification of $\mathscr{B}\left(\mathscr{M}^{(n)}\right)$ with $\mathcal{N}_{n}(\mathscr{B}(\mathscr{M}))$.) It is a consequence of Theorem 2.8 in [1] that $\pi\left(\underline{M}_{i}^{*}\right)$ is unitarily equivalent to $M_{i}^{*\left(n_{i}\right)} \oplus W_{i}$ with $\sigma\left(W_{i}\right) \subseteq \partial D$ and $0<n_{i} \leqslant \infty$. Indeed, the $*$-representation $\pi: \mathscr{B}(\mathscr{M}) \rightarrow \mathscr{B}(\mathscr{K})$ gives rise to a $*$-representation $\pi_{i}$ : $\mathscr{B}\left(\mathscr{M}_{i}\right) \rightarrow \mathscr{B}(\mathscr{K})$ defined by $\pi_{i}(A)=\pi(1 \otimes \cdots \otimes A \otimes \cdots \otimes 1), A \in \mathscr{B}\left(\mathscr{M}_{i}\right)$; and Theorem 2.8 in [1] is directly applicable. The assertion $\sigma\left(W_{i}\right) \subseteq \partial D$ is essentially a consequence of the fact that $\lambda-M_{i}$ is Fredholm for every $\lambda$ in $D$.

Remark. With $V$ having the same meaning as in Definition 1.3, Theorem 2.5 enables us to write $T_{i}$ as $T_{i}=V^{*} \pi\left(\underline{M}_{i}{ }^{*}\right) V=V^{*} U_{i}{ }^{*}\left(M_{i}{ }^{*\left(n_{i}\right)} \oplus W_{i}\right) U_{i} V$, where $U_{i}$ is some unitary operator. The assumption $\sigma\left(T_{i}\right) \subset D$ can further be utilized to conclude that $T_{i}=V^{*} U_{i}{ }^{*} M_{i}{ }^{*\left(n_{i}\right)} U_{i} V$ (see the proof of Theorem 2.3 in [1]).

We now state the analog of Theorem 3.1 in [1]. 
THEOREM 2.6. Let $\mathscr{M}, \kappa, T$ have the same meaning as in Theorem 2.5. Suppose also that $\frac{1}{\kappa}$ extends to a holomorphic function on a neighborhood of $D^{m} \times D^{m}$. Then the following are equivalent.

(i) $\frac{1}{\kappa}\left(T, T^{*}\right) \geqslant 0$.

(ii) There exist a Hilbert space $\mathscr{K}$ and a $*$-representation $\pi: \mathscr{B}(\mathscr{M}) \rightarrow \mathscr{B}(\mathscr{K})$ with $\pi(1)=1$ such that $T$ extends to $\pi\left(\underline{M}^{*}\right)$, and where $\pi\left(\underline{M}_{i}^{*}\right)$ is unitarily equivalent to $M_{i}^{*\left(n_{i}\right)} \oplus W_{i}$ with $\sigma\left(W_{i}\right) \subseteq \partial D$ and $0<n_{i} \leqslant \infty(1 \leqslant i \leqslant m)$.

Proof. That (i) implies (ii) follows from Theorem 2.5. The proof that (ii) implies (i) is a result of

$$
\frac{1}{\kappa}\left(T, T^{*}\right)=V^{*} \frac{1}{\kappa}\left(\pi\left(\underline{M}^{*}, \underline{M}\right)\right) V=V^{*} \pi\left(\frac{1}{\kappa}\left(\underline{M}^{*}, \underline{M}\right)\right) V .
$$

Indeed, $\frac{1}{\kappa}\left(\underline{M}^{*}, \underline{M}\right) \geqslant 0$ as follows by considering a sequence of polynomials converging to $\frac{1}{\kappa}$ uniformly on compact subsets of a neighborhood of $D^{m} \times D^{m}$ (see (4)) and using Lemma 2.4. Since $\pi$ is a $*$-representation, $\pi\left(\frac{1}{\kappa}\left(\underline{M}^{*}, \underline{M}\right)\right)$ is positive as well.

3. Applications. The classical Hardy space $\left(H^{2}\left(\mu_{1}\right)\right)$ and Bergman space $\left(H^{2}\left(\mu_{2}\right)\right)$ are among a series of regular analytic model atoms $H^{2}\left(\mu_{n}\right)$ over $D$ discussed in [2]. We recall that $H^{2}\left(\mu_{n}\right)$ is the completion of polynomials in $L^{2}\left(D, \mu_{n}\right)$ and the measures $\mu_{n}$ are defined in a recursive fashion starting with the normalized Lebesgue measure on $\partial D$. The kernel $\kappa_{\mu_{n}}$ of $H^{2}\left(\mu_{n}\right)$ is given by $\kappa_{\mu_{n}}(z, w)=1 /(1-z w)^{n}$. The following is the analog of Theorem 1.10 in [2].

THEOREM 3.1. Let $T$ be a tuple of $m$ commuting operators on a Hilbert space $\mathscr{H}$. Also, let $M_{i}$ denote multiplication by $z$ on $H^{2}\left(\mu_{\kappa_{i}}\right)$. Then the following are equivalent.

(i) There exist a Hilbert space $\mathscr{K}$ and $a *$-representation

$$
\pi: \mathscr{B}\left(H^{2}\left(\mu_{k_{1}}\right) \otimes \cdots \otimes H^{2}\left(\mu_{k_{m}}\right)\right) \rightarrow \mathscr{B}(\mathscr{K})
$$

with $\pi(1)=1$ such that $T$ extends to $\pi\left(\underline{M}^{*}\right)$, and where $\pi\left(\underline{M}_{i}^{*}\right)$ is unitarily equivalent

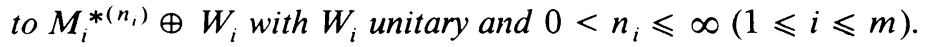

(ii) $\left(\prod_{i=1}^{m}\left(1-z_{i} w_{i}\right)^{p_{i}}\right)\left(T, T^{*}\right) \geqslant 0$ for all $p_{i}$ such that $0 \leqslant p_{i} \leqslant k_{i}$.

Proof. The proof that (i) implies (ii) is similar to the proof that (ii) impliles (i) in Theorem 2.6. We now prove (ii) implies (i). Suppose $\left(\prod_{i=1}^{m}\left(1-z_{i} w_{i}\right)^{p_{i}}\right)\left(T, T^{*}\right) \geqslant 0$ for all $p_{i}$ such that $0 \leqslant p_{i} \leqslant k_{i}$. Note that for $0<\mathrm{s}<1$,

$$
\begin{aligned}
\left(\prod_{i=1}^{m}\right. & \left.\left(1-z_{i} w_{i}\right)^{k_{i}}\right)\left(s T, s T^{*}\right)=\left(\prod_{i=1}^{m}\left(1-s^{2} z_{i} w_{i}\right)^{k_{i}}\right)\left(T^{*}, T^{*}\right) \\
& =\left(\prod_{i=1}^{m}\left(1-z_{i} w_{i}+\left(1-s^{2}\right) z_{i} w_{i}\right)^{k_{i}}\right)\left(T, T^{*}\right) \\
& =\left(\prod_{i=1}^{m}\left(\sum_{j=0}^{k_{i}}\left(\begin{array}{c}
k_{i} \\
j
\end{array}\right)\left(1-s^{2}\right)^{k_{i}-j} w_{i}^{k_{i}-j}\left(1-z_{i} w_{i}\right)^{j} z_{i}^{k_{i}-j}\right)\right)\left(T, T^{*}\right) .
\end{aligned}
$$

Expanding the bracketed function and appealing to (3) repeatedly, it is clear that the last expression is a positive operator. Since each $T_{i}$ is a contraction, $\sigma\left(s T_{i}\right) \subset D$ for each $i$. It follows by Theorem 2.5 that there exist a Hilbert space $\mathscr{K}_{0}$ and a 
*-representation $\pi_{0}: \mathscr{B}\left(H^{2}\left(\mu_{k_{1}}\right) \otimes \cdots \otimes H^{2}\left(\mu_{k_{m}}\right)\right) \rightarrow \mathscr{B}\left(\mathscr{K}_{0}\right)$ with $\pi_{0}(1)=1$ such that $s T$ extends to $\pi_{0}\left(\underline{\tilde{M}}^{*}\right)$. But then by Theorem 1.4 we have that $\left[p_{i j}\left(\underline{M}^{*}, \underline{M}\right)\right] \geqslant 0$ in $\mathscr{N}_{n}\left(\mathscr{B}\left(H^{2}\left(\mu_{k_{1}}\right) \otimes \cdots \otimes H^{2}\left(\mu_{k_{m}}\right)\right)\right)$ implies $\left[p_{i j}\left(s T, s T^{*}\right)\right] \geqslant 0$ in $\mathscr{B}\left(\mathscr{H}^{(n)}\right)$. Letting $s \rightarrow 1$, we see that $\left[p_{i j}\left(\underline{M}^{*}, \underline{M}\right)\right] \geqslant 0$ implies $\left[p_{i j}\left(T, T^{*}\right)\right] \geqslant 0$. So by Theorem 1.4 again, there exist a Hilbert space $\mathscr{K}$ and a $*$-representation

$$
\pi: \mathscr{B}\left(H^{2}\left(\mu_{k_{1}}\right) \otimes \cdots \otimes H^{2}\left(\mu_{k_{m}}\right)\right) \rightarrow \mathscr{B}(\mathscr{K})
$$

with $\pi(1)=1$ such that $T$ extends to $\pi\left(\underline{M}^{*}\right)$. As before, $\pi\left(\underline{M}_{i}^{*}\right)$ has the form $M_{i}{ }^{*\left(n_{i}\right)} \oplus W_{i}$ with $\sigma\left(W_{i}\right) \subseteq \partial D$ and $0<n_{i} \leqslant \infty(1 \leqslant i \leqslant m)$. Combining the proof of Theorem 2.8 in [1] with the fact that each $M_{i}$ is essentially unitary (see Proposition 1.3 in [2]), it is not difficult to show that each $W_{i}$ is unitary.

COROLlaRY. Let $T$ be a tuple of $m$ commuting operators on a Hilbert space $\mathscr{H}$. Let, for each $i, M_{i}$ denote the multiplication operator $M$ on the Hardy space $H^{2}\left(\mu_{1}\right)$. Then the following are equivalent.

(i) There exist a Hilbert space $\mathscr{K}$ and $a *$-representation

$$
\pi: \mathscr{B}\left(H^{2}\left(\mu_{1}\right) \otimes \cdots \otimes H^{2}\left(\mu_{1}\right)\right) \rightarrow \mathscr{B}(\mathscr{K})
$$

with $\pi(1)=1$ such that $T$ extends to $\pi\left(\underline{M}^{*}\right)$, where $\pi\left(\underline{M}_{i}^{*}\right)$ is unitarily equivalent to $M_{i}^{*\left(n_{i}\right)} \oplus W_{i}$ with $W_{i}$ unitary and $0<n_{i} \leqslant \infty(1 \leqslant i \leqslant m)$.

(ii) $T_{1}, \ldots, T_{m}$ are $m$ commuting contractions such that $T=\left(T_{1}, \ldots, T_{m}\right)$ has a regular unitary dilation [13].

Proof. In both (i) and (ii), the necessary and sufficient conditions are

$$
\left(\prod_{i=1}^{m}\left(1-z_{i} w_{i}\right)^{p_{i}}\right)\left(T, T^{*}\right) \geqslant 0
$$

for $0 \leqslant p_{i} \leqslant 1(1 \leqslant i \leqslant m)$.

REMARK 1 . In case $m=1$, the results here reduce to those in $[1,2]$.

REMARK 2. Theorems 2.5, 2.6, and 3.1 allow an infinite number of interesting possibilities. Thus if $M_{1}$ denotes the unilateral shift and $M_{2}$ denotes the Bergman shift, the necessary and sufficient conditions for $\left(T_{1}, T_{2}\right)$ to extend to $\left(\pi\left(\underline{M}_{1}^{*}\right), \pi\left(\underline{M}_{2}^{*}\right)\right)$ with $\pi(1)=1$ are

$$
\left(\left(1-z_{1} w_{1}\right)^{p_{1}}\left(1-z_{2} w_{2}\right)^{p_{2}}\right)\left(T_{1}, T_{2}, T_{1}^{*}, T_{2}^{*}\right) \geqslant 0
$$

for $0 \leqslant p_{1} \leqslant 1,0 \leqslant p_{2} \leqslant 2$. If $\sigma\left(T_{i}\right) \subset D$ for $i=1,2$, then the relevant conditions are

$$
\left(\left(1-z_{1} w_{1}\right)\left(1-z_{2} w_{2}\right)^{2}\right)\left(T_{1}, T_{2}, T_{1}^{*}, T_{2}^{*}\right) \geqslant 0 .
$$

The modification of Theorem 3.1 and in particular of conditions (ii) there when some of the operators $T_{i}$ have their spectra inside $D$ is obvious and will not be stated.

REMARK 3 . The unitary part in the representation of $\pi\left(\underline{M}_{i}^{*}\right)$ in Theorem 3.1 can be removed to get $T_{i}=V^{*} U_{i}^{*} M_{i}^{*\left(n_{i}\right)} U_{i} V$ by requiring that $T_{i}^{m}$ tend to zero strongly as $m \rightarrow \infty$. 
4. Commuting normal extension. In [2], Agler showed that for $T \in \mathscr{B}(\mathscr{H})$, the following are equivalent.

(i) $\|T\| \leqslant 1$ and $T$ is subnormal.

(ii) $(1-z w)^{n}\left(T, T^{*}\right) \geqslant 0$ for all $n \geqslant 1$.

(In this connection, the statement on p. 212 in [2] of a result of Sz.-Nagy needs to be corrected.)

In view of our work in the previous sections and the above result, it is natural to look for conditions for $T=\left(T_{1}, \ldots, T_{m}\right)$ to have a commuting normal extension, that is, for $m$ commuting normal operators $N_{1}, \ldots, N_{m}$ to exist so that $T$ extends to $N=\left(N_{1}, \ldots, N_{m}\right)$. In what follows, we may assume without any loss of generality that for each $i,\left\|T_{i}\right\|=\left\|N_{i}\right\|$. The following theorem is a consequence of the solution of the Hausdorff Moment Problem in several dimensions.

THEOREM 4.1. Let $T$ be a tuple of $m$ commuting operators on a Hilbert space $\mathscr{H}$. Then the following are equivalent.

(i) $\left\|T_{i}\right\| \leqslant 1$ for $1 \leqslant i \leqslant m$ and $T$ has a commuting normal extension $N$.

(ii) For any choice of nonnegative integers $k_{i}(1 \leqslant i \leqslant m)$,

$$
\left(\prod_{i=1}^{m}\left(1-z_{i} w_{i}\right)^{k_{i}}\right)\left(T, T^{*}\right) \geqslant 0 \text {. }
$$

Proof. That (i) implies (ii) can be checked easily by utilizing $N_{i} N_{j}=N_{j} N_{i}$, $N_{i} N_{j}^{*}=N_{j}^{*} N_{i}$, and $\left\|N_{i}\right\|=\left\|T_{i}\right\|$ for all $i$ and $j$. Conversely suppose (ii) holds. Let $N=\{0,1,2, \ldots\}$, and for $p, n \in N^{m}$ such that $n_{i} \geqslant p_{i}(1 \leqslant i \leqslant m)$, write

$$
|p|=p_{1}+\cdots+p_{m}, \quad\left(\begin{array}{l}
n \\
p
\end{array}\right)=\left(\begin{array}{l}
n_{1} \\
p_{1}
\end{array}\right)\left(\begin{array}{l}
n_{2} \\
p_{2}
\end{array}\right) \cdots\left(\begin{array}{l}
n_{m} \\
p_{m}
\end{array}\right) .
$$

Then conditions (ii) can be written as $\sum_{p}(-1)^{|p|}\left(\begin{array}{l}k \\ p\end{array}\right) T^{* p} T^{p} \geqslant 0$ for all $k \in N^{m}$. Let $s \in N^{m}$ and let $u$ be any vector in $\mathscr{H}$. Then we obviously have

$$
\sum_{p}(-1)^{|p|}\left(\begin{array}{l}
k \\
p
\end{array}\right)\left\langle T^{p+s} u, T^{p+s} u\right\rangle_{\mathscr{H}} \geqslant 0 \text { for all } k \in N^{m} .
$$

Here $p+s=\left(p_{1}+s_{1}, \ldots, p_{m}+s_{m}\right)$. Now define a function $\varphi_{u}$ from $N^{m}$ to the real line $\mathbf{R}$ by $\varphi_{u}(n)=\left\langle T^{n} u, T^{n} u\right\rangle_{\mathscr{H}}=\left\|T^{n} u\right\|_{\mathscr{H}}^{2}$. Then the conditions just derived can be expressed as $\Delta_{1}^{k_{1}} \Delta_{2}^{k_{2}} \cdots \Delta_{m}^{k_{m}} \varphi_{u}(s) \geqslant 0$ for any $s, k \in N^{m}$, where the difference operator $\Delta_{i}$ acts on $\varphi_{u}$ by $\Delta_{i} \varphi_{u}(s)=\varphi_{u}(s)-\varphi_{u}\left(s_{1}, \ldots, s_{i}+1, \ldots, s_{m}\right)$. (In other words, the function $\varphi_{u}$ is "completely monotone" on $N^{m}$.) It follows then by a result of T. N. Hildebrandt and I. J. Schoenberg [6] that there exists a positive measure $\mu_{u}$ on $[0,1]^{m}$ such that

$$
\left\langle T^{* s} T^{s} u, u\right\rangle_{\mathscr{H}}=\left\|T^{s} u\right\|_{\mathscr{H}}^{2}=\varphi_{u}(s)=\int_{[0,1]^{m}} x^{s} d \mu_{u}(x) .
$$

If for any measurable set $A \subset[0,1]^{m}$ and $u \in \mathscr{H}$, we define $\langle\rho(A) u, u\rangle_{\mathscr{H}}=\mu_{u}(A)$, then the above implies that $T^{* s} T^{s}=\int_{[0,1]^{m}} x^{s} d \rho_{u}(x)$. A simple change of variables and an application of a result of A. Lubin [8] now yield that $T$ has a commuting normal extension. That each $T_{i}$ is a contraction is obvious, and the proof is complete. 
We conclude this section by trying to unify the ideas implicit in the preceding analysis. First a few definitions.

Definition 4.2. Let $S$ be a semigroup with identity and with involution *. A function $\psi: S \rightarrow \mathscr{B}(\mathscr{H})$ is called positive definite if $\sum_{s, t}\left\langle\psi\left(s^{*} t\right) f(t), f(s)\right\rangle_{\mathscr{H}} \geqslant 0$ for all functions $f: S \rightarrow \mathscr{H}$ such that $f$ has finite support.

Definition 4.3. Let $S$ be as in Definition 4.2, and let $\mathscr{H}$ be a Hilbert space. A function $\psi: S \rightarrow \mathscr{B}(\mathscr{H})$ is said to be $*$-dilatable if there exist a Hilbert space $\mathscr{K}$, a *-preserving semigroup homomorphism $\varphi: S \rightarrow \mathscr{B}(\mathscr{K})$, and a bounded linear operator $V: \mathscr{H} \rightarrow \mathscr{K}$ such that $\psi(s)=V^{*} \varphi(s) V, s \in S$.

THEOREM 4.4. Let $T$ be a tuple of $m$ commuting operators on $\mathscr{H}$. Let $N=$ $\{0,1,2, \ldots\}$ and $\tilde{N}$ be the semigroup $N^{m} \times N^{m}$ with multiplication as coordinatewise addition and with the involution * defined by $(p, q)^{*}=(q, p), p, q \in N^{m}$. Let $\psi$ : $\tilde{N} \rightarrow \mathscr{B}(\mathscr{H})$ be defined by $\psi(p, q)=T^{* q} T^{p}$. Then the following are equivalent.

(i) $T$ has a commuting normal extension.

(ii) $\psi$ is positive definite on $\tilde{N}$.

(iii) $\psi$ is *-dilatable on $\tilde{N}$.

(iv) Multi-dimensional Halmos-Bram conditions [7] hold.

(v) Multi-dimensional Embry conditions [8] hold.

(vi) For any $u \in \mathscr{H}$, the function $\varphi_{u}: N^{m} \rightarrow \mathbf{R}$ defined by $\varphi_{u}(p)=\left\|S^{p} u\right\|_{\mathscr{H}}^{2}$, where $S_{i}=\alpha T_{i}(\alpha \neq 0)$ are so chosen that $\left\|\alpha T_{i}\right\| \leqslant 1(1 \leqslant i \leqslant m)$, is completely monotone.

Proof. The equivalence of (i) and (vi) can be deduced from the proof of Theorem 4.1. That (ii) implies (iii) can be proved by specializing a result of F. H. Szafraniec [11] to the semigroup $\tilde{N}$ and then applying a result of Sz.-Nagy [12]. The rest of the implications are either easy to prove or well known.

We remark that in the one-dimensional case, a parallel version of the above results with the semigroup $\tilde{N}$ replaced by the additive group of complex numbers and the map $\psi$ replaced by the map $\eta(s)=e^{-s^{*} T^{*}} e^{s T}(s \in \mathbf{C})$, can be found in [5]. We see from Theorem 4.4 that for $m$ contractions $T_{1}, \ldots, T_{m}$ on $\mathscr{H}$, conditions (ii) in Theorem 4.1 are equivalent to requiring the function $\psi$ as defined in Theorem 4.4 to be positive definite. It is interesting to note that the partial fulfillment of these conditions leads to the kind of results stated in Theorem 3.1.

ACKNOWLEDGMENTS. I wish to thank John B. Conway for his constant guidance throughout the preparation of this work. Thanks are also due to W. Szymanski who pointed out to me the one-dimensional version of a portion of Theorem 4.4.

\section{REFERENCES}

1. J. Agler, The Arveson extension theorem and coanalytic models, Integral Equations Operator Theory 5 (1982), 608-631.

2. , Hypercontractions and subnormality, J. Operator Theory 13 (1985), 203-217.

3. N. Aronszajn, Theory of reproducing kernels, Trans. Amer. Math. Soc. 68 (1950), 337-404.

4. W. B. Arveson, Subalgebras of $C^{*}$-algebras, Acta Math. 123 (1969), 141-224.

5. J. Bram, Subnormal operators, Duke Math. J. 22 (1955), 75-94.

6. T. N. Hildebrandt and I. J. Schoenberg, On linear functional operators and the moment problem for a finite interval in one or several dimensions, Ann. of Math. 34 (1933), 317-328. 
7. T. Ito, On the commutative family of subnormal operators, J. Fac. Sci. Hokkaido Univ. 14 (1958), $1-15$.

8. A. Lubin, Weighted shifts and commuting normal extension, J. Austral. Math. Soc. 27 (1979), 17-26.

9. R. Narasimhan, Several complex variables, Univ. of Chicago Press, Chicago and London, 1971.

10. J. R. Ringrose, Compact non-self-adjoint operators, Van Nostrand Reinhold, London, 1971.

11. F. H. Szafraniec, Dilations on involution semigroups, Proc. Amer. Math. Soc. 66 (1977), 30-32.

12. B. Sz.-Nagy, Extensions of linear transformations in Hilbert space which extend beyond this space, Appendix to F. Riesz and B. Sz.-Nagy, Functional Analysis, Ungar, New York, 1960.

13. B. Sz.-Nagy and C. Foias, Harmonic analysis of operators on Hilbert space, North-Holland, Amsterdam, 1970.

Department of Mathematical Sciences, Indiana University-Purdue University at IndianapoLis, 1125 East 38Th Street, P. O. Box 64\%, Indianapolis, Indiana 46223 